\title{
The Application of New Materials Technology in Basketball
}

\author{
Yiqi Song* \\ Department of Physical Education, North China Institute of Science and Technology, Hebei 065201, China \\ Email: songyq1981@yeah.net
}

\begin{abstract}
With the rapid development of modern materials technology, application of new materials in basketball has become more widely. By using new materials technology in basketball, not only athletics' performance can be improved and make the game more enjoyable, but also athletics can be protected from sports injuries. Based on the status of basketball development, applications of the advanced materials in Basketball will be described in this report.
\end{abstract}

Keywords-new Material, basketball, development

\section{INTRODUCTION}

Basketball was an indoor sport invented by Dr. James Naismith, a physical education teacher in Springfield YMCA training school (later renamed Springfield College). When he found a solution to the problem that outdoor sport like football, baseball cannot be conducted in December 1891. Now, basketball has been born for more than 100 years. With the improvement of basketball rules and athletes techniques, the technology of associated shoes, clothing, and other products has been developed greatly. Thus, not only the game become more enjoyable, but also athletics can be protected from accidental injuries.

\section{SPORT SHOES}

Playing basketball is intense that players constantly change direction on the pitch, change the speed. The impetus comes mainly from the player when he stamp the ground to get his reaction. And in this process, the athlete's low limbs would bear the brunt of some power. In addition, athletes are also very vulnerable to external damage when they have intense campaign confrontations. In order to protect the basketball players from accidental injury, manufacturers carefully select materials and design shoe.

In terms of external support, manufacturers usually design a generous support box pasted on the bottom. The commonly selected material is fiber polymer that can withstand a certain impact, avoiding a wide range of heel movement. Combined with holding structure design, the shoes can effectively minimize sports injury. Cushioning properties is an important index of basketball shoes. When athletes are competing for a rebounding or jumping, their bodies withstand the equivalent of five times their own weight impact. Therefore, the sole material is very important. Manufacturers usually will choose PHYLON (also known as MD) as a basketball shoe's mid-bottom The characteristic of the material is its light-weight, good elasticity, good shock performance and easy cleaning. Further, the German Evonik Degussa group developed nylon-12 compound, which were mainly used in the production of high-grade sports shoes, making basketball shoes the road lighter and steadier. Also, nylon-12 compound has good strength, strong rigidity, elasticity and protecting the foot joint from damage. At the same time, the material has good chemical resistance, which is hardly affected by temperature. In order to better protect the basketball athlete and reduce of the impact caused by jumping and running, NIKE Company used air cushion technology injecting high pressure gas though synthetic rubber layer in their early product Air-sole. With relatively balanced shock ability, stable performance and response speed, this technology has wide applicability and was applied in various kinds of sport shoes. Later, Nike has launched the Air Max, Zoom Air, Tuned Air, Total Air and other products.

\section{BASKETBALL WEAR}

Basketball game is featured with energy consumption, intensity, much sweat, long time, complicated technique, and much body movement, etc. Players have to conduct violent activities, such as running, jumping, throwing, turning-around, sudden stop, breakthrough in the game, etc. Besides, the game is about 90 minutes with great physical expenditure, and the players will become sweat much too, so the demands for the basketball wear are mainly on moisture-wicking, quick-drying, thermal diffusivity, permeability and so on.

At the early stage of NBA, the only requirement for the manufacturer of the basketball wear made by the officials is solid and durable, so the cloth used is Dacron, cotton and satin, and did not place too much on comfort. As time went by, fabric technology has been improved, which gave the players new exceptions on the materials of sports wear, 60' clothing sponsor of NBA, MacGregor Sand-Knit used mix materials of cotton-Dacron to replace the traditional satin, which improved and enhanced the comfort of the wear together with "Mesh Design" [1]. Du Pont Company launched a novel hydroscopic and sweat-discharge polyester fiber named "CoolMax" in the United States in 1986, and the clothes made of it will almost dry (98\%) 30min after being washed; later on, textile raw materials suppliers all over the world began to invest heavily to develop related products with moisture absorption and 
sweat releasing capabilities[2]. Adidas and Reebok also became the clothing sponsors of NBA after 2000, and "Better sweat releasing and Better permeability" are the effort targets of the two manufacturers; all the team uniforms of NBA have been upgraded by 2010, and the weight of the new uniforms only accounted $60 \%$ of former ones, Cli-macool fabric with better moisture absorption and sweat releasing capabilities was used. In the all-star game of the same year, the tight-fitting shirt TECHFIT specially made by Adidas Company is not only breathable, but also can prevent foul play like pulling the shirt that the defensive players make, which can make athletes faster ${ }^{2}$. Another giant of sportswear manufacturer Nike also launched its own product in the same period, such as Nike F. I. T and Nike Sphere series, which can meet different requirements of perspiration, heat preservation and anti-sunburn and others for different sports.

\section{BASKETBALL}

Spalding is a world famous basketball brand, and they will develop and design new products for NBA every year. And their raw materials for top products are provided by the world famous leather manufacturer GANASON, who selects the natural skin from the back of the cattle and use it for making advanced basketball products. This kind of basketball will help the players with more control on shots, and make players feel dry but not sticky when there is sweat on the surface, with moderate bounce height and easily to manipulate. But these products are expensive, and difficult to maintain, only for Interior wood floors, and this basketball is currently used in NBA. In addition to make customized basketballs for NBA, the Company also makes balls for other national teams and Leagues, like ZK micro-fiber basketballs. This kind of balls is characterized by smooth, sweat-absorbent and non-slip, abrasion, mildew-resistant, as well as easy to maintain, etc., and CBA also uses this kind of balls.

With the development of environmental protection concept, lots of sports equipment suppliers start using synthetic leather to make basketball like PU and PVC synthetic materials. Basketballs made from $P U$ are featured with waterproof, wear-resistant, mildew-resistant and are suitable for interior and exterior conditions. Though basketballs made from PVC are slightly worse in hand-touch and resistance to low temperature compared with PU synthetic leather, but better in waterproof, abrasion, mildew-resistant and other capabilities than the basketballs made from PU materials, and has an advantage in price, which is the main ingredient of low-end basketball products.

\section{ACKNOWLEDGEMENTS}

The author thank Dr. Jian He for his advice on this paper and Ningbo Han for his language edit.

\section{CORRESPONDING AUTHOR}

Yiqi Song, Email: songyq1981@yeah.net, Tel: 13700349458

\section{REFERENCES}

[1] Tsan Bing. Development Trends of US Sportswear from the Perspective of Professional Sports. Technology Horizons. (2012), Issue 14.

[2] Zhang Xiaoyan. Application of New Materials in Sportswear. Guangxi Textile Technology. (2009), Issue 1. 